\title{
The IncRNA UCA1 promotes proliferation, migration, immune escape and inhibits apoptosis in gastric cancer by sponging anti-tumor miRNAs
}

\author{
Chao-Jie Wang ${ }^{\dagger}$, Chun-Chao Zhư ${ }^{\dagger}$, Jia Xu, Ming Wang, Wen-Yi Zhao, Qiang Liu, Gang Zhao and Zi-Zhen Zhang ${ }^{*}$ (D)
}

\begin{abstract}
Background: UCA1 is a long non-coding RNA which was found overexpressed in various human cancers including gastric cancer (GC). It is identified that UCA1 promotes GC cells proliferation, migration and invasion, however, the role of UCA1 during the processes of immune escape is still not unclear.

Methods: We collected 40 paired GC and non-tumor tissue samples. The level of UCA1 in GC and control tissue samples were determined by in situ hybridization and qRT-PCR. Cell viability was determined by MTT assay. GC cells' migration capacities were examined by transwell assay. To understand the roles of UCA1 during immune escape, wildtype or UCA1 KO GC cells co-cultured with peripheral blood mononuclear cells or cytokine-induced killer cells in vitro. Mouse model was used to examine the function of UCA1 in vivo.

Results: UCA1 promoted GC cells proliferation and migration, and inhibit apoptosis. UCA1 repressed miR-26a/b, miR-193a and miR-214 expression through direct interaction and then up-regulated the expression of PDL1.

UCA1-KO GC cells could induce a higher IFNy expression when co-cultured with peripheral blood mononuclear cells (PBMCs), and have a lower survival rate when co-cultured with cytokine-induced killer (CIK) cells in vitro.

UCA1-KO GC cells formed smaller tumors, had higher miR-26a, -26b, -193a and - 214 level, reduced cell proliferation and increased apoptosis in xenograft mouse model.
\end{abstract}

Conclusions: UCA1 overexpression protected PDL1 expression from the repression of miRNAs and contributed to the GC cells immune escape. UCA1 could serve as a potential novel therapeutic target for GC treatment.

Keywords: IncRNA, UCA1, Gastric cancer, miRNA, PDL1

\section{Introduction}

As the fourth most common cancer worldwide, gastric cancer (GC) is still incurable and induces about 700, 000 mortalities each year [1-4]. What is worse, nonresectable or metastatic GC is associated with poor prognosis, and systemic chemotherapeutic approaches provide minimal benefit. Thus, novel biomarkers for improving GC, early diagnosis, prognostic evaluation and tumor grading is urgently needed.

\footnotetext{
* Correspondence: zhangzizhen@renji.com

Chao-Jie Wang and Chun-Chao Zhu equal contributors

Department of Gastrointestinal Surgery, Ren Ji Hospital, School of Medicine,

Shanghai Jiao Tong University, No. 160 Pu Jian Road, Shanghai 200127,

China
}

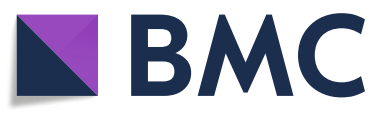

(c) The Author(s). 2019 Open Access This article is distributed under the terms of the Creative Commons Attribution 4.0 International License (http://creativecommons.org/licenses/by/4.0/), which permits unrestricted use, distribution, and

reproduction in any medium, provided you give appropriate credit to the original author(s) and the source, provide a link to the Creative Commons license, and indicate if changes were made. The Creative Commons Public Domain Dedication waiver (http://creativecommons.org/publicdomain/zero/1.0/) applies to the data made available in this article, unless otherwise stated. high throughput sequencing, scientists realized that the most part of human genome is transcribed to nonprotein coding RNAs, which indicates that a large group of RNA regulators is dedicated to regulating a relatively small amount of effectors [5-7]. Among the newly discovered RNA elements, long non-coding RNAs (lncRNAs) have been identified to function as key regulators of diverse cellular processes, such as development, differentiation, and cell fate as well as disease pathogenesis $[8,9]$. IncRNAs can serve as signal mediators, molecular decoys and scaffold or enhancers of transcription and what is intriguing is that a large group of lncRNAs function as competing endogenous 
RNA (ceRNA) that regulate gene expression through absorbing miRNAs [10].

Until now, different groups have screened the expression profile of lncRNAs in GC tumors and found several disordered lncRNAs relates to GC carcinogenesis such as PVT1, H19, LNC00152 and so on [11-13]. However, the underling mechanisms are still not well understood.

UCA1 (urothelial carcinoma associated 1) is a lncRNA that was first identified in human bladder carcinoma, whose expression is found to be up-regulated in many other cancers till now [14]. In gastric cancer, overexpressed UCA1 has been found in tumor tissues compared with paired non-cancerous samples, and the function of UCA1 have been confirmed to contribute to the cancer cell migration, invasion and drug resistance $[15,16]$.

In this study, we examined the level of UCA1 in different GC subtype tissue samples and unveiled the mechanism of how UCA1 modulates GC cells proliferation, migration and immune escape.

\section{Materials and methods}

\section{Cell lines and human gastric cancer tissues}

Human gastric cancer cell line AGS, SGC-7901, BGC823, MGC-803 and SNU-1 were obtained from National Infrastructure of Cell Line Resource (Beijing, China). Primary stomach epithelial cells from two healthy individuals were purchased from Abace-biology (Beijing, China) (www.abace-biology.com/primary-cell.htm) All the cells were maintained in humidified incubator at $37^{\circ} \mathrm{C}$ in a CO2 incubator in RPMI 1640 medium supplemented with $10 \%$ fetal bovine serum (FBS) and $1 \%$ penicillin-streptomycin.

A total of 40 primary gastric adenocarcinoma tumor tissues and corresponding adjacent non-tumorous gastric tissue samples were obtained between 2013 and 2015 at Ren Ji Hospital, Shanghai Jiao Tong University. A part of each tissue samples was subject to formalin fixation and paraffin-embedment. And another part of each tissue samples was stored in a refrigerator at $-80^{\circ} \mathrm{C}$. All the 40 paired tumor and non-tumor tissues were subjected to following study including immunoblotting, RNA extraction and qRT-PCR.

\section{Plasmid construction}

Full length of 2314 UCA1 sequence was cloned into pCR3.1 vector to construct the UCA1 overexpression vector. For dual-luciferase assay, full length of UCA1 was cloned into pmirGLO vector, following firefly luciferase coding region. A $621 \mathrm{bp}$ segment of PDL1 3'UTR was cloned into pmirGLO vector to form PDL1 3'UTR luciferase reporter vector.

For UCA1 knockdown construction, two guide RNAs targeting the promoter region of UCA1 were constructed into lenti-guide-puro vector (Addgene: 52963), named
pUCA1-KD. For UCA1 knockout cell establishment, AGS or SGC-7901 cells were co-transfected with pUCA1-KD and plentiCas9-Blast (Addgene: 52962) followed by selection for more than 5 days with blasticidin $(4 \mu \mathrm{g} / \mathrm{ml})$ and puromycin $(2 \mu \mathrm{g} / \mathrm{ml})$ for 1 week.

\section{RNA real-time RT-qPCR}

Quantitive RT-PCR analysis was used to determine the relative expression level of mRNAs and miRNAs. Total RNA was extracted from clinical samples and cells, using Trizol Reagent (Invitrogen, Carlsbad, CA, USA) according to the manufacturer's instructions. The RNA samples were treated using TURBO DNA-free kit to remove the DNA contamination and then the DNA contamination was assessed by agarose gel. RevertAid First Strand cDNA Synthesis Kit (Thermo Scientific) was employed for the reverse-transcription, and the level of specific RNAs were determined using $\mathrm{SYBR}^{\circ}$ Green real-time PCR kit (Invitrogen, Carlsbad, CA, USA) with GADPH as loading control. The level of $\beta$-actin was also quantified to confirm the relative expression of UCA1.

The expression level of miRNAs was detected by TaqMan miRNA RT-Real Time PCR. Single-stranded cDNA was synthesized by using TaqMan MicroRNA Reverse Transcription Kit (Applied Biosystems, Foster City, CA, USA) and then amplified by using TaqMan Universal PCR Master Mix (Applied Biosystems, Foster City, CA, USA) together with miRNA-specific TaqMan MGB probes (Applied Biosystems, Foster City, CA, USA). The U6 snRNA was used for loading control. The level of small nucleolar RNA, C/D box 58B (RNU58B) was also quantified to confirm the relative expression of miRNAs. Each sample in each group was measured in triplicate and the experiment was repeated at least three times for the detection of miRNAs.

\section{RNA in situ hybridization}

Slides were deparaffinized and rehydrated through immersion in xylene and an ethanol gradient and then digested with proteinase $\mathrm{K}(20 \mu \mathrm{g} / \mathrm{mL})$ for $20 \mathrm{~min}$ at $37^{\circ} \mathrm{C}$. Slides were firstly fixed in $4 \%$ paraformaldehyde for $5 \mathrm{~min}$ at room temperature, and then dehydrated by immersion in an ethanol gradient and air dried; slides were prehybridized using DIG Easy Hyb (Roche, Mannheim, Germany) at $50^{\circ} \mathrm{C}$ for $1 \mathrm{~h}$. The $10 \mathrm{pmol}$ digoxin-labeled UCA1 DNA probe was denatured in hybridization buffer at $95^{\circ} \mathrm{C}$ for $2 \mathrm{~min}$ and then chilled on ice. The UCA1 probe was diluted in $250 \mu \mathrm{L}$ pre-warmed in hybridization buffer. Each sample was covered with $50-100 \mu \mathrm{L}$ diluted probe and incubated in a humidified hybridization chamber at $50^{\circ} \mathrm{C}$ overnight. Slides were washed twice in $50 \%$ formamide in $4 \times \mathrm{SSC}$ at $37^{\circ} \mathrm{C}$ for $30 \mathrm{~min}$, and then washed three times in $2 \times \mathrm{SSC}$ at $37^{\circ} \mathrm{C}$ for $15 \mathrm{~min}$. After twice washing with maleic acid buffer containing Tween- 
20 (MABT), slides were blocked using blocking buffer (Roche, Mannheim, Germany) at room temperature for $30 \mathrm{~min}$, the blocking buffer was drained off, and the samples were incubated with 1:250 diluted Anti-DigoxigeninAP Fab fragments (Roche, Mannheim, Germany) at $37^{\circ} \mathrm{C}$ for $1 \mathrm{~h}$. After washing twice in MABT and once in detection solution $(0.1 \mathrm{M}$ Tris- $\mathrm{HCl}, 0.1 \mathrm{M} \mathrm{NaCl}, \mathrm{pH} 9.5)$, the slides were stained with freshly diluted NBT/BCIP detection solution (Roche, Mannheim, Germany) and incubated at $37^{\circ} \mathrm{C}$ for $30 \mathrm{~min}$. Slides were washed in PBS twice, air dried for $30 \mathrm{~min}$, and then mounted with Eukitt quickhardening mounting medium (Sigma Aldrich, St. Louis, MO, USA). Images were obtained using a microscopy (Leica DM2000 LED) and a digital camera (Leica DMC 2900). Three different random images were captured for each sample at $400 \times$ magnification and the relative density of UCA1 signal was quantified by Image J. The results were analyzed using paired t-test and $p<0.05$ was statistically significant.

\section{Fluorescence in situ hybridization (FISH)}

AGS cells were rinsed by PBS and then fixed in $4 \%$ formaldehyde for $10 \mathrm{~min}$ at RT. Further, the cells were permeabilized in PBS containing 0.5\% Triton X-100 and $5 \mathrm{mM}$ VRC(New England Biolabs Inc., USA) on ice for $10 \mathrm{~min}$, then washed with PBS. After rinsed once in $2 \times$ SSC, hybridization was carried out using FAM labelled UCA1 DNA probe at $37^{\circ} \mathrm{C}$ for $12 \mathrm{~h}$. After three times washing by $2 \times \mathrm{SSC}$, one of the candidate miRNAs was detected by incubation with Cy5 labelled miRNA specific probe at $37^{\circ} \mathrm{C}$ for $12 \mathrm{~h}$. After nucleus staining by DAPI, images were captured by confocal microscopy (Leica TCS SP8) at 630× magnification.

\section{RNA antisense purification (RAP)}

Cell extracts were incubated with biotin labelled UCA1 probe at $4{ }^{\circ} \mathrm{C}$ for $2 \mathrm{~h}$, with biotin labelled sequence scrambled DNA oligo as control. Hybridized material was captured with magnetic streptavidin beads (Thermo Fisher Scientific). Beads were subjected to RNA extraction followed by RT-qPCR to quantify miRNAs levels.

\section{Cell apoptosis assay}

The number of apoptotic cells were evaluated by assaying for annexin V. Cells were stained with FITC labeled Annexin V antibody and PI (BioLegend), and then cells were analyzed using flow cytometry. Results were analyzed using Flowjo software (Ashland).

\section{Cell proliferation assay}

AGS and SGC-7901 cells were seeded in 96-well plates at density of $5 \times 103$ cells per well in RPMI 1640 culture, and allowed to attach overnight. pCR3.1-UCA1 was transfected into cells for $48 \mathrm{~h}$, with empty vector as control. Twenty microliters MTT (5 mg/ml) (Sigma, St. Louis, MO, USA) were added into each well and the cells were incubated for further $4 \mathrm{~h}$. The absorbance was recorded at A570nm with a 96-well plate reader after the DMSO addition.

\section{In vitro migration assay}

Indicated cells were deprived of serum overnight, treated with mitomycin-C, seed into the upper chamber at density of $1 \times 105 /$ well $(8 \mu \mathrm{m}$ pore size; BD Bioscience). The chemoattractant in the lower chamber was filled with medium supplemented with 10\% FBS. After $12 \mathrm{~h}$, the lower chambers cells were fixed with $4 \%$ paraformaldehyde and then stained with crystal violet. The migrated cells were counted in 5 randomly different fields with an inverted microscope. The experiments were performed in triplicate wells and each experiment was performed in triplicate.

\section{Generation mouse xenograft model}

The experiments including animals were approved by Ren Ji Hospital Animal Care and Use Committee. Female severe combined immunodeficient (SCID) beige mice were purchased from Charles River Laboratories at age 8 weeks with a weight between $20 \mathrm{~g}$ and $25 \mathrm{~g}$. A total of $5 \times 10^{6}$ SGC-7901 cells in $100 \mu \mathrm{L}$ PBS together with an equal volume of Matrigel basement membrane matrix were injected subcutaneously into the shoulder to establish a human GC cell xenograft model.

\section{Immunoblotting}

Protein samples were boiled in SDS/ $\beta$-mercaptoethanol sample buffer, and $20 \mu \mathrm{g}$ of each sample was loaded into each lane of $4-12 \%$ polyacrylamide gels. After separation by electrophoresis, the proteins in the gels were transferred onto PVDF membranes (Amersham Pharmacia Biotech, St. Albans, Herts, UK). The membrane was incubated with rabbit anti-CDK6 polyclonal antibody (ab151247, Abcam, Cambridge, MA, USA) or rabbit anti-EZH2 monoclonal antibody (ab191080, Abcam, Cambridge, MA, USA) or rabbit anti-PDL1 polyclonal antibody (ab205921, Abcam, Cambridge, MA, USA) or rabbit anti-TAK1 polyclonal antibody (ab109526, Abcam, Cambridge, MA, USA) or rabbit anti-KRAS polyclonal antibody (ab180772, Abcam, Cambridge, MA, USA), or rabbit anti-FGF9 polyclonal antibody (ab71395, Abcam, Cambridge, MA, USA) or mouse anti- $\beta$ actin monoclonal antibody (Santa Cruz Biotechnology Inc., Santa Cruz, CA, USA) over night at $4{ }^{\circ} \mathrm{C}$. The specific protein-antibody complex was detected by using horseradish peroxidase conjugated goat anti-rabbit or rabbit antimouse IgG. Detection by the chemiluminescence reaction was carried using the ECL kit (Pierce, Appleton, WI, USA). The $\beta$-actin signal was used as a loading control. 


\section{Immunohistochemistry}

Paraffin-embedded sections were firstly deparaffinized and then incubated with rabbit polyclonal anti-Ki-67 (ab15580, Abcam, Cambridge, MA, USA) or rabbit polyclonal antiPDL1 (ab205921, Abcam, Cambridge, MA, USA) primary antibody at $4{ }^{\circ} \mathrm{C}$ overnight. After three times wash by TBST, the sections were then incubation with HRP conjugated goat anti-mouse or goat anti-rabbit secondary antibody. The sections were washed by TBST for three times and the signal was detected using DAB Substrate kit following the manufacture's instruction. Images were obtained using a microscopy (Leica).

\section{CIK cells preparation}

The peripheral blood was obtained from patients using heparin as the anticoagulant. The peripheral blood mononuclear cells (PBMCs) were isolated by Ficoll-Conray density gradient centrifugation, as described previously [17].The PBMCs $(2.0 \times 106 / \mathrm{ml})$ were plated onto 6-well dishes and cultured with RPMI 1640 in the presence of human IFNy $(1.0 \times 106 \mathrm{U} / \mathrm{L})$; recombinant human interleukin $2(5.0 \times$ $105 \mathrm{U} / \mathrm{L}) ; 10 \%$ inactivated human serum; $25 \mathrm{mM}$ HEPES; and $2 \mathrm{mMl}$-glutamine. After $24 \mathrm{~h}$, monoclonal antibody against CD3 $(100 \mu \mathrm{g} / \mathrm{L}$, Antibody Diagnostic Inc., New York, NY, USA) and interleukin-1 alpha $(1.0 \times 105 \mathrm{U} / \mathrm{L})$ were added. 2 days after incubation, the cells were cultured in medium without IFN $\gamma$ and the medium was replaced every 3 days.

\section{Cytotoxicity activity analysis}

For the cytotoxicity analysis, UCA1-KO and control GC cells were used as the target cells, while CIK cells were used as the effector cells. The effector and target cells were co-cultured in triplicate at ratios of 10 : 1, 20:1 and 40:1 with the wells containing just effector cells or target cells as controls. The cells were maintained in a humidified incubator at $37^{\circ} \mathrm{C}$ in $5 \%$ $\mathrm{CO} 2$ for $24 \mathrm{~h} .100 \mu \mathrm{l}$ medium from the co-cultured cells was mixed with $20 \mu \mathrm{l}$ CCK- 8 solutions, and then incubated for another $4 \mathrm{~h}$. The optical density (OD) was recorded at $450 \mathrm{~nm}$ for each well by a microplate reader. The survival $(\%)=($ effector target cell mixtureeffector group)/target cell*100\%.

\section{Statistical analysis}

Two-tailed Student's t test was used to calculate statistical significance between two comparator groups. The differences of lncRNA expressions between paired tissue sample was evaluated with Wilcox matched pairs signed ranks test. The correlation analysis was analyzed by $\chi^{2-}$ analysis. ALL the data were analyzed by using SPSS Statistical Package version 16. The survival times of different groups of patients were analyzed using the Kaplan-
Meier method. A $p$ value $<0.05$ was considered as statistically significant.

\section{Results}

Increased UCA1 was found in patients with GC and related with poor prognosis in patients with intestinal GC UCA1 is a lncRNA whose expression is found to related to the initiation and progression of many kinds of cancers. To explore its function in GC, we first analyzed the expression of UCA1 in GC tissues and noncancerous control tissues by using two published microarray datasets, GSE54129 (111 GC patients and 21 control) and GSE65801 (32 paired GC and noncancerous tissues). We found a significantly overexpressed UCA1 in patients with GC (Fig. 1a). We also collected 40 paired GC and noncancerous tissue samples (21 intestinal, 13 diffused and 7 mixed) and examined the UCA1 level by qRTPCR. We found UCA1 level only up-regulated in the intestinal GC subtype tissues significantly (Fig. 1b). To further confirm up-regulation of UCA1 in GC tissues and identify its subcellular location, we detected the UCA1 in GC and control tissue sections using in situ hybridization (ISH). As shown in Fig. 1c, dense UCA1 signal only was found in GC tissues and mainly existed in the cytoplasm. After quantification and statistical analysis, the ISH results indicated that UCA1 was overexpressed in GC tissues. Finally, we analyzed the data reported by Szász AM et al. (320 intestinal GC, 241 diffused GC and 32 mixed), and found intestinal GC patients with higher UCA1 level have a poor overall survival, suggesting UCA1 has a potential oncogenic role during the carcinogenesis of GC(Fig. 1d) [18].

\section{UCA1 functioned as an oncogene promoted proliferation, migration and inhibit apoptosis in GC cells}

To further explore the biological function of UCA1 in the GC cells, we first constructed UCA1 overexpression vector and established UCA1 overexpression GC cell lines (Fig. 2a). As shown in Fig. 2b, GC cells with overexpressed UCA1 have significantly increased cell viability. Meanwhile, we constructed UCA1 knockdown vector which expresses two guide RNAs targeting the promoter region of UCA1 and then established UCA1 knockdown cell lines (Fig. 2c, d). The UCA1 promoter region knockout was confirmed by genotyping and sequencing(Additional file 1: Figure S1). As shown in Additional file 1: Figure S1A, The wildtype PCR product is $1705 \mathrm{bp}$ which between bands of $1500 \mathrm{bp}$ and $2000 \mathrm{bp}$ of the marker lane. Meanwhile, the expected UCA1 knockdown PCR products are around $1103 \mathrm{bp}$ which located between bands of $1000 \mathrm{bp}$ and $1500 \mathrm{bp}$ of the marker lane.

We found significantly reduced viability and increased apoptosis in the UCA1 knockdown cells (Fig. 2e, f). 
A

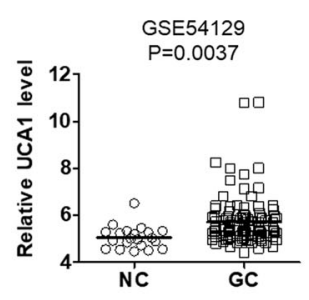

C



D

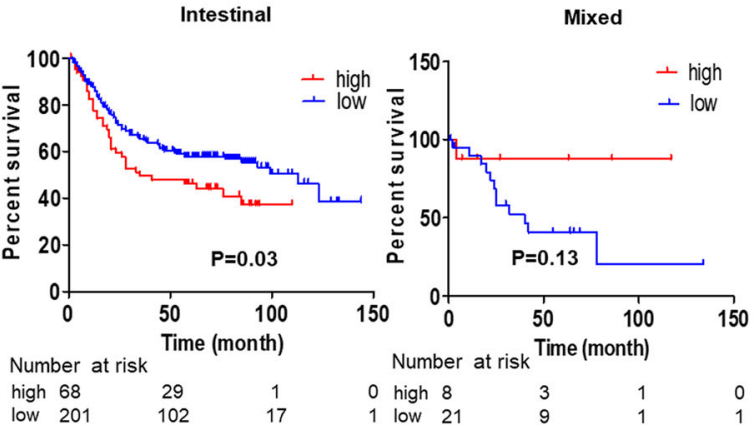

B
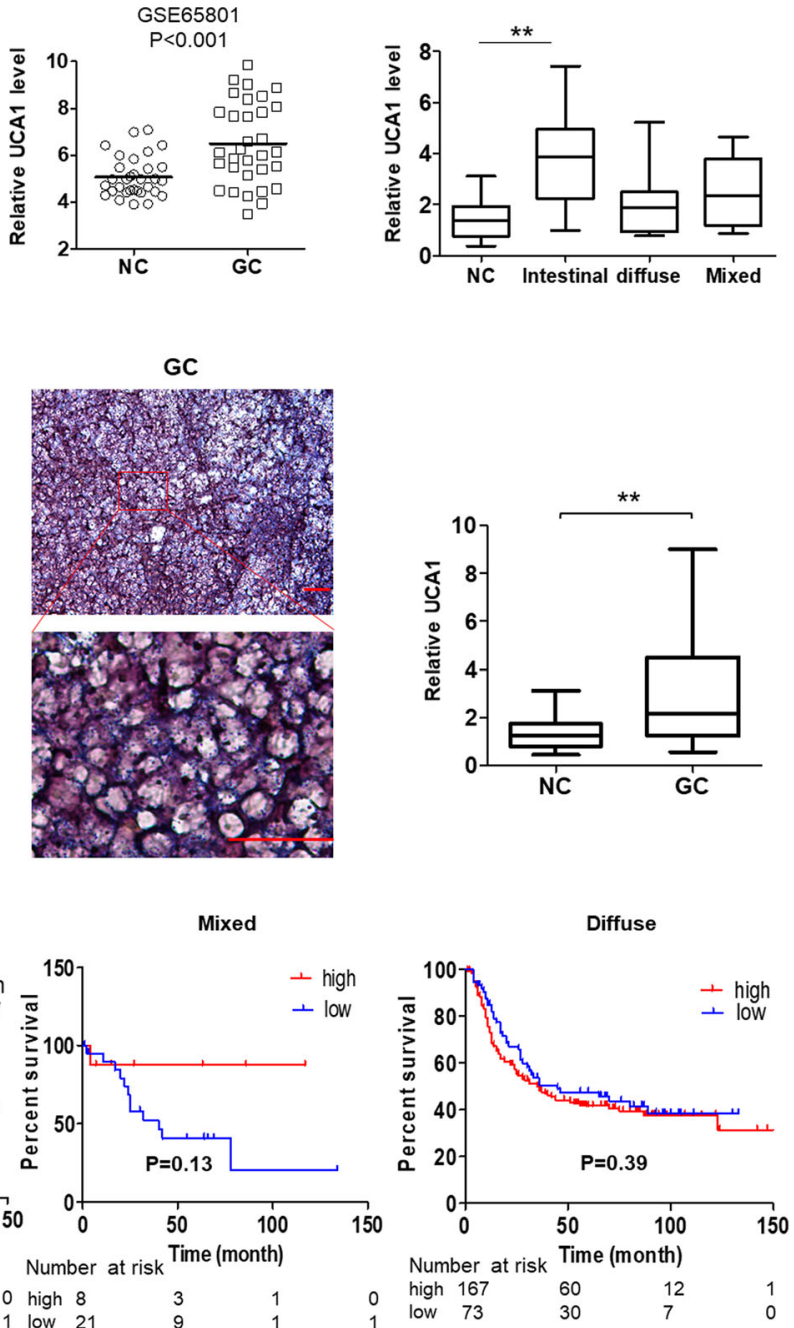

Fig. 1 dysregulated UCA1 was found in GC tissues and relates to worse prognosis. a The UCA1 level from two microarray datasets (GSE54129and GSE65801) was analyzed by student's t-test. $P<0.05$ was considered statistically significant. b The UCA1 level in 40 paired gastric adenocarcinoma and corresponding adjacent non-tumorous gastric tissue was detected by qPCR. Results were exhibited according to Lauren classification and analyzed by One-way ANOVA. ${ }^{* *} p<0.01$. c Representative images of in situ hybridization showing UCA1 expression within GC and control tissues. The relative density of UCA1 signal was quantified by Image J, and the significant was determined by student's t-test. Scale bar, $100 \mu M$. $\mathbf{d}$ The survival data containing 320 intestinal GC, 241 diffused GC and 32 mixed GC were analyzed by Kaplan-Meier analysis and $p<0.05$ was considered statistically significant

Subsequently, examined by transwell assay, UCA1 overexpression GC cells exhibited increased motility and UCA1 knockout GC cells have reduced migration cell numbers (Fig. 2g, h).

\section{UCA1 interacted with $\mathrm{miR}-26 \mathrm{a} / \mathrm{b}$ and regulated the expression of EZH2 and CDK6}

Accumulating evidence indicates that lncRNAs can exert "sponge-like" effects on various miRNAs, which subsequently inhibits miRNA-mediated functions [19]. So, we firstly analyzed the potential of interaction between UCA1 and miRNAs. We found
miR-26a and -b may bind with UCA1 directly, and this binding was subsequently confirmed by dualluciferase assay (Fig. 3a, b), RAP assay (Additional file 3: Figure S3A) and FISH (Additional file 3: Figure S3B). Meanwhile, when UCA1 was overexpressed in the GC cells, the expression of endogenous miR-26a and -b was significantly downregulated Fig. $3 \mathrm{c}$ and $\mathrm{d}$. We also detected the protein level of two miR-26 targets EZH2 and CDK6 that were confirmed by other researchers [20, 21]. As shown in the right panel of Fig. 3c and d, the level of EZH2 and CDK6 was significantly increased in the UCA1 
A

C
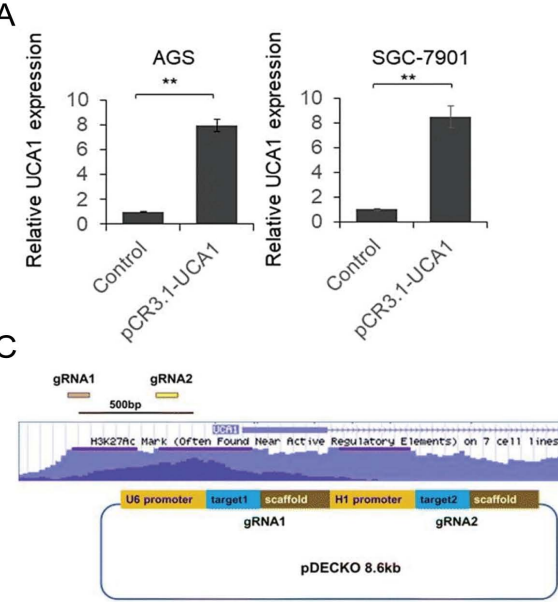

$E$



B
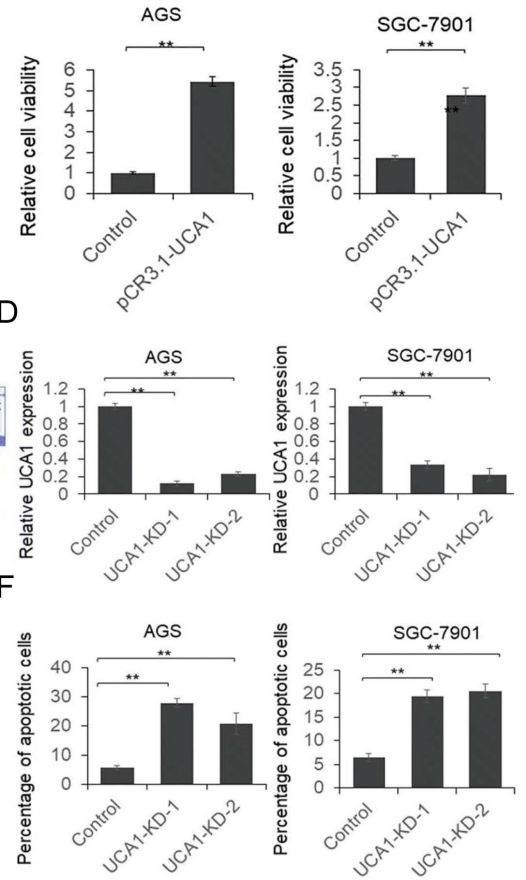

G
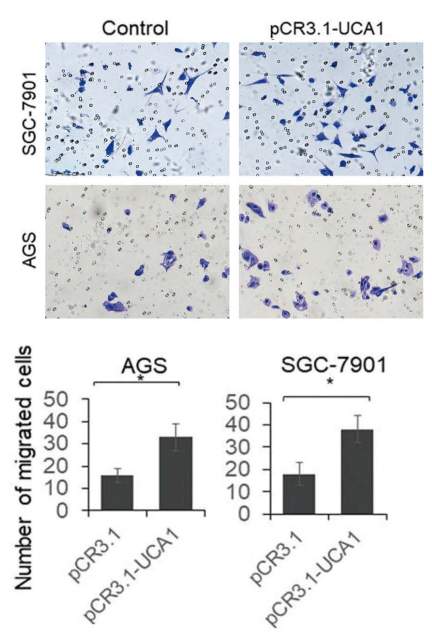

$\mathrm{H}$

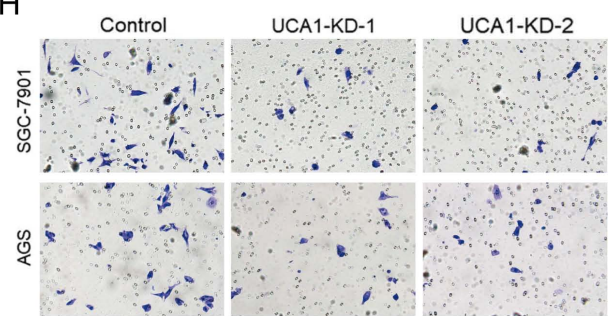

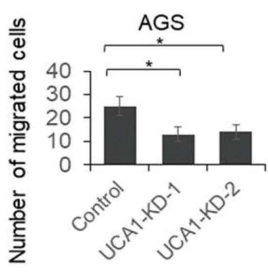



Fig. 2 UCA1 functions as an onco-IncRNA promotes GC cells proliferation, migration, and inhibits apoptosis. a UCA1 overexpression GC cells were successfully established. b MTT assay was used to determine the cell viability of UCA1 overexpression and control GC cells. c Schematic diagram indicates the UCA1 knock-out vector design. Two guide RNAs targeting the promoter region of UCA1 were co-expressed by one plasmid. $\mathbf{d}$ UCA1 level was successfully reduced by co-transfecting UCA1-KD vector and Cas9 expression vector in two GC cells. e MTT assay to determine the cell viability of UCA1-KD GC cells. $\mathbf{f}$ Apoptosis assay. UCA1-KD or control GC cells were incubated with FITC labeled Annexin V antibody and then stained by PI. The percentage of apoptosis cells were determined by flow cytometry. $\mathbf{g}$ and $\mathbf{h}$ cells were deprived of serum overnight, treated with mitomycin$C$ and introduced into the upper chamber of the Transwell. Cells that migrated to the lower chambers were fixed with $4 \%$ paraformaldehyde and then stained with crystal violet. Crystal violet-stained cells were counted in 5 randomly different fields with an inverted microscope. Results were analyzed by student's t-test and $p<0.05$ was considered statistically significant. ${ }^{*} p<0.05$, ${ }^{* *} p<0.01$

overexpression cells, suggesting the function of miR26 was repressed. To further determine miR-26a and $-\mathrm{b}$ level in vivo, we detected miR-26a and -b expression using qRT-PCR. We found decreased miR-26a in all the GC tissues and decreased miR- 26b in intestinal GC tissues (Fig. 3e). We also found a significant strong negative correlation between UCA1 and miR-26b (Fig. 3e).

To verify that UCA1 regulate EZH2 and CDK6 expression through sponging miR-26a and miR-26b, 
A

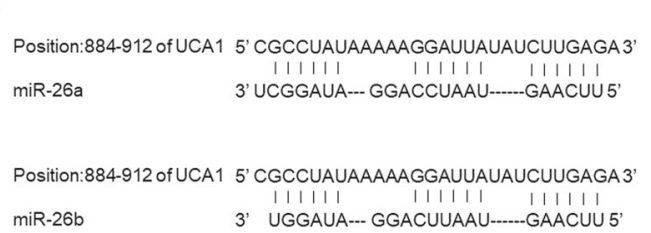

C
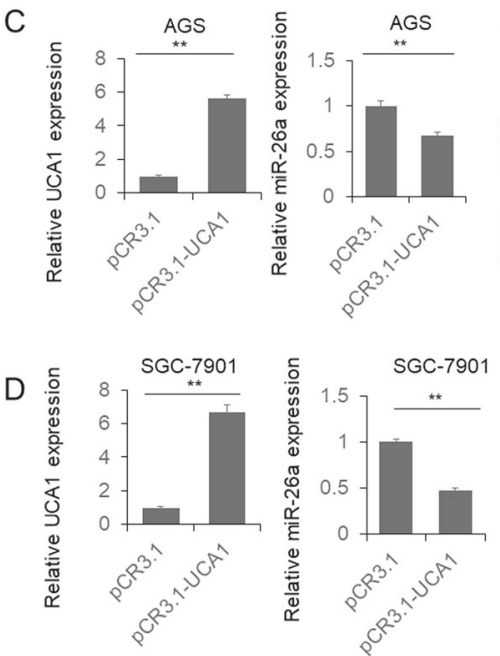

E
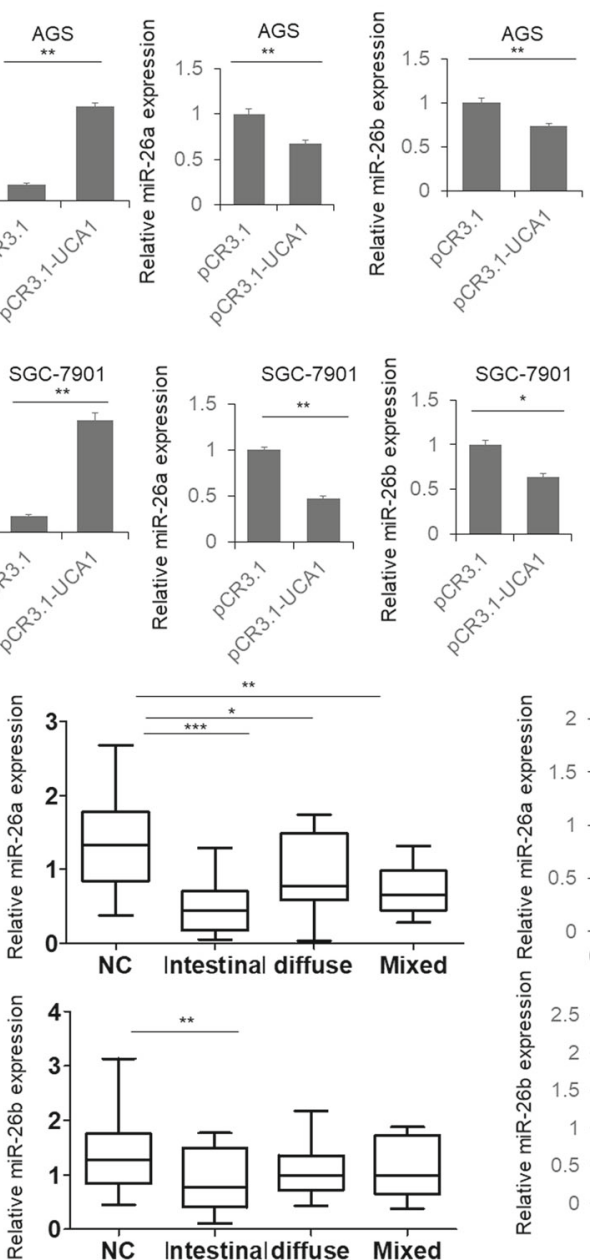
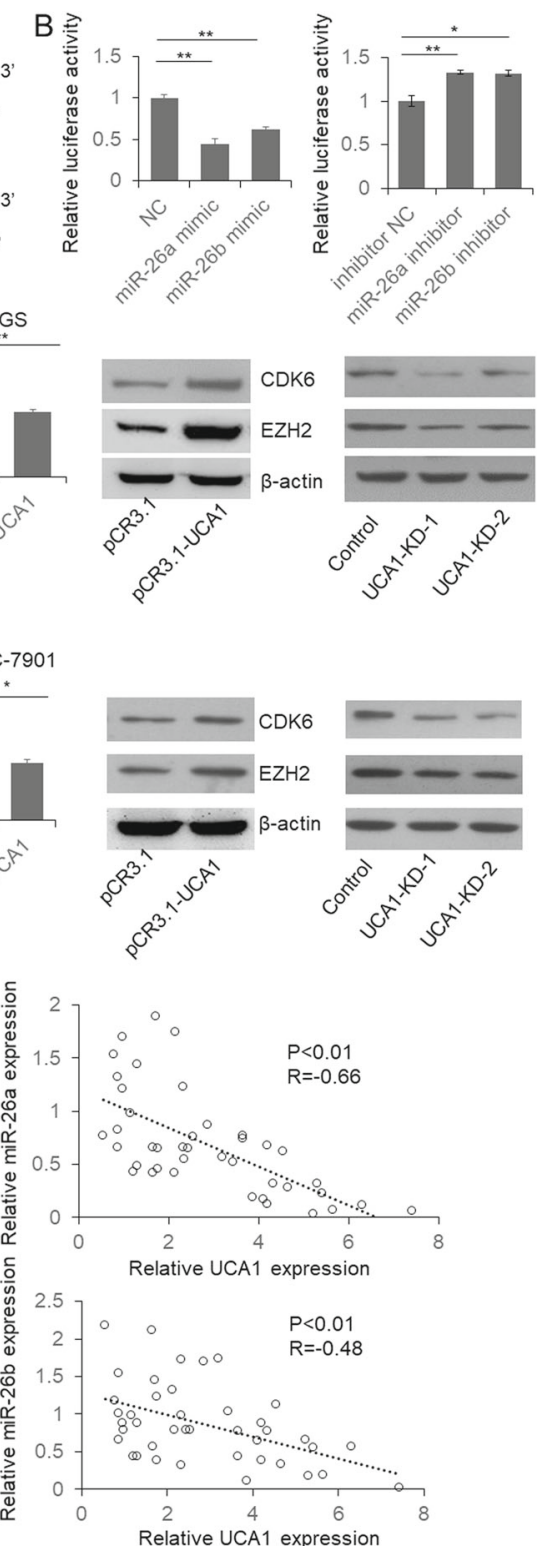

Fig. 3 UCA1 sponges miR-26a/b and promote the expression of miR-26a/b target genes. a predicted interaction between miR-26a/b and UCA1. b dual luciferase assay. HEK293T cells transfected with UCA1 3'UTR reporter vector and miR-26a/b mimic or inhibitor. Relative luciferase activity was detected $48 \mathrm{~h}$ post interaction. $\mathbf{c}$ and $\mathbf{d}$ miR-26a and $-\mathrm{b}$ levels were repressed in UCA1 overexpression GC cells, and two miR-26a and $-\mathrm{b}$ target genes are up-regulated. e the level of miR-26a and -b in 40 paired GC and adjacent non-tumor control samples was determined by qRTPCR. The Correlation between UCA1 and miR-26a or $-b$ was analyze by correlation analysis

we first quantified the EZH2 and CDK6 protein level in tumour and control tissues by immunoblotting. Results indicated that both EZH2 and CDK6 protein were increased in GC tissues (Additional file 2: Figure S2A) and negatively correlated with UCA1 level(Additional file 2: Figure S2B and C). Meanwhile, in the UCA1 siRNA treated cells, EZH2 and CDK6 expressions were repressed, which can be rescued by $\mathrm{miR}-26 \mathrm{a} / \mathrm{b}$ antagonists(Additional file 2 : Figure S2D). These results indicated that UCA1 regulated EZH2 and CDK6 expression through sponging $\mathrm{miR}-26 \mathrm{a} / \mathrm{b}$.

\section{UCA1 directly interact with miR-214 and miR-193a}

In addition, we also found miR-214 and -193 have the potential to be 'sponged' by UCA1 (Fig. 4a), and both were reported function as tumor suppressors $[22,23]$. Dual luciferase assay was processed to confirm the interaction between miRNAs and UCA1. As shown in Fig. 4b, luciferase activities were repressed 
A

\begin{tabular}{|c|c|}
\hline Position:2146-2166 of UCA 1 & $\begin{array}{l}\text { 5' AUACGGACACGCGU.--GACAGGA 3' } \\
|1| 1|||||1| 1|1| 1 \mid\end{array}$ \\
\hline $\operatorname{miR}-214$ & 3' CGUGUC_GUUCACAUCUGUCCGU 5' \\
\hline Position:1739-1764 of UCA 1 & 5' CCGUCCAGGUGGCCUGC-.-AGGAGCCAA 3' \\
\hline $\operatorname{miR}-193 a$ & 3' AGUAG-...-A GCGGGCGUUUCUGGGU 5' \\
\hline
\end{tabular}

C

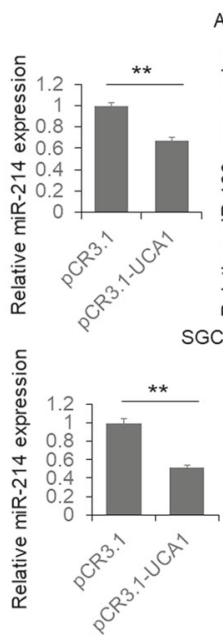

F

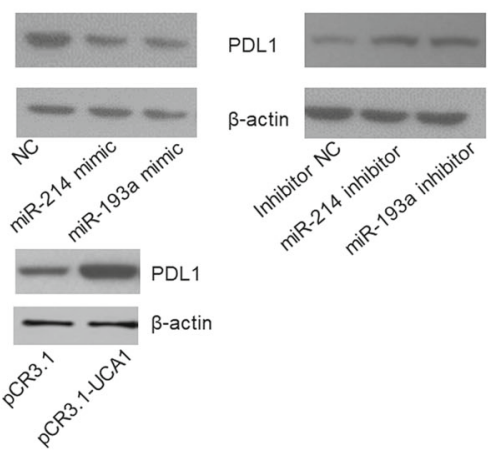

E
B

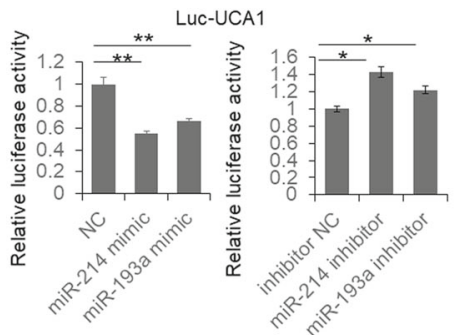

D

$$
\begin{aligned}
& \text { Position:2407-2413 of PDL13'UTR 5' UUCUUUGUGUGAAUUACAGGCAA3' } \\
& \text { miR-214 3' CGUGUCGUUCACAUCUGUCCGU } 5 \\
& \text { Position 1914-1920 of PDL1 3'UTR 5' UGUUUGCUCAAAAGGAGACCCAU 3' } \\
& \text { miR-193a 3'AGUAGAGCGGGCGUUUCUGGGU 5' }
\end{aligned}
$$
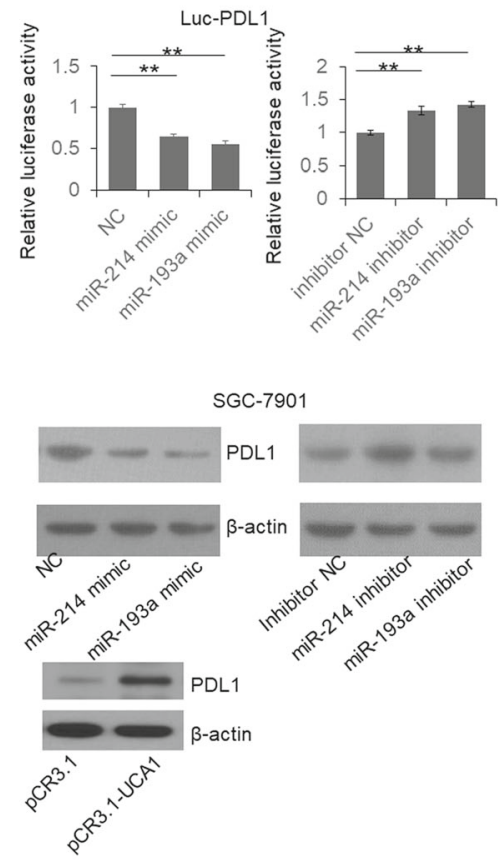

Fig. 4 UCA1 interacts with miR-193a and miR-214, and then modulate PDL1 expression. a predicted interaction between UCA1 and miR-193a or miR-214. $\mathbf{b}$ dual luciferase assay. HEK293T cells were co-transfected with reporter vector using full sequence of UCA1 as 3'UTR of firefly luciferase, and miRNAs mimics or inhibitors. The luciferase activity was detected $48 \mathrm{~h}$ post transfection. $\mathbf{c}$ The relative miR-193a and - 214 expression was determined by qRT-PCR. $\mathbf{d}$ predicted interaction between PDL1 and miR-193a or miR-214. e dual luciferase assay. HEK293T cells were cotransfected with PDL1 3'UTR reporter vector and the mimic or inhibitor of miR-193a or miR-214 for $48 \mathrm{~h}$. Luciferase activities were detected and results were analyzed by student's t-test. f miR-193a and miR-214 repress endogenous PDL1 expression

by miR-193a and - 214 mimics, and up-regulated by miR-193a and - 214 inhibitors, meanwhile, the level of miR-193a and-214 was reduced in UCA1 overexpression cells (Fig. 4c). In the GC tissues, both of miR-193 and miR-214 were reduced and negatively correlated with UCA1 level (Additional file 4: Figure $\mathrm{S} 4 \mathrm{~A}$ ), indicating the direct interaction between miRNAs and UCA1. The direct interaction between UCA1 and miR-193 or miR-214 was confirmed by RAP assay (Additional file 3: Figure S3A) and FISH (Additional file 3: Figure S3B) in GC cells.
UCA1 regulated PDL1 expression through sponging miR214 and miR-193a

PDL1 is an important member of the B7 family molecules which can interact with PD-1 to inhibit the T-cell activation, induce apoptosis of effector T cells, and finally impair the anti-tumor immunity [24, 25]. Increasing evidences indicated that PDL1 overexpression in many human cancers is significantly associated with tumor progression and patient's prognosis [26, 27]. Predicted by online bioinformatics tools, we surprisingly found miR-214 and -193a target sites in the 3'UTR of PDL1 Mrna (Fig. 4d). Subsequent dual- 
luciferase assay indicated that miR-214 and -193a can repress luciferase activity by targeting the 3'UTR of PDL1(Fig. 4e). Meanwhile, in the gastric cancer cells, the endogenous PD-L1 expression was also modulated by miR-214 and -193a (Fig. 4f). In intestinal GC tissues, PD-L1 expression was found increased and positively correlated with UCA1 level(Additional file 4: Figure S4B).

To further determine whether UCA1 knock out can induce PDL1 downregulation in GC cells by sponging miRNAs, and subsequently alleviate $\mathrm{T}$ cell suppression, we first analyzed the PDL1 in the UCA1 knock out GC cells by western blot and flow cytometry. We found a significant reduced PDL1 expression and left shift peak of the UCA1-KO GC cells (Fig. 5a,b). Meanwhile, the peak left shift can partially rescued by antagonists for miR-193a and miR-214 suggesting the cell surface PDL1 level was regulated by UCA1-miR-193a/214 axis.

\section{UCA1 regulated PDL1 expression and modulate anti- tumour immune response}

Subsequently, we evaluated if modulation of PDL1 expression on GC cell lines could alter PHA-stimulated activation of healthy donor PBMC as measured by the released supernatant IFNY level. As shown in Fig. 5c, IFNY release by PHA stimulated PBMC was partially suppressed by the AGS-NC or SGC7901-NC cells when compared with

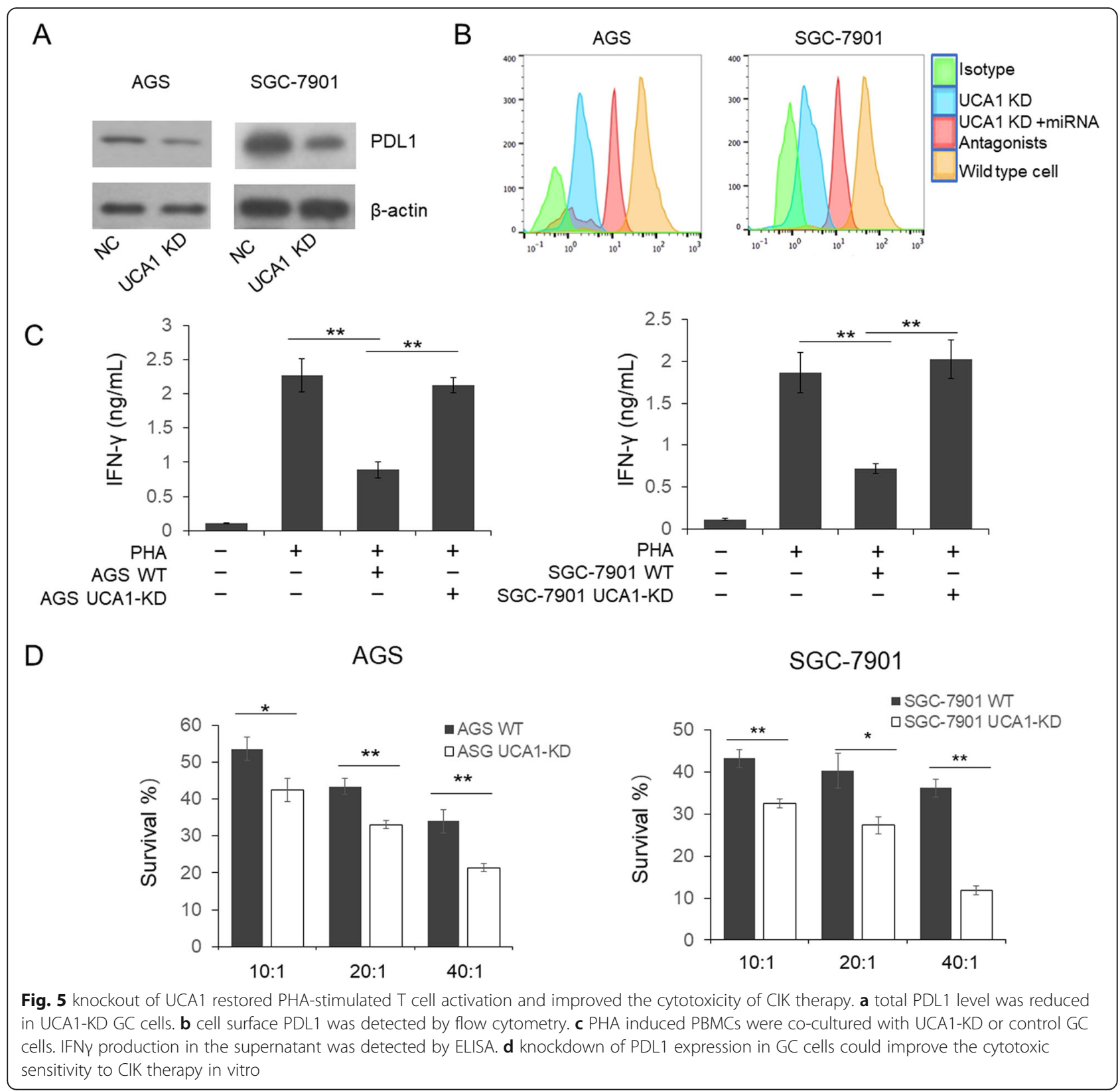


PHA-stimulated PBMC alone suggesting a significant PDL1 mediated immune suppressive effect. When PBMCs co-cultured with UCA-KO GC cells, the IFNy levels were increased, indicated that PDL1 downregulation induced by UCA1 knock out alleviated the immune suppressive effect. Subsequently, we analyzed the cytotoxicity of CIK cells on both AGS and SGC-7901 cells when the effector-target ratio was in the range 10:1 to 40:1. As shown in Fig. 5d, the cancer cell survival rate was obviously related to the effector-target ratio, and decreased with increase of the effector-target ratio. The cytotoxic activity of the CIK cells against AGS-UCA1-KO cells was markedly higher than that of AGS-NC cells under the same conditions (Fig. $5 \mathrm{~d}$, $p<0.05)$, and the same in SGC-7901 cells.

In order to explore the effect of UCA1 knockout in the regulation of tumor growth in vivo, we further examined the function of UCA1 using AGS cell subcutaneous xenograft mouse model. The AGS-NC and AGSUCA1-KO cells were injected subcutaneously into SCID mice. The tumor volumes were measured every 7 days for 28 days. As shown in Fig. 6a, knockout of UCA1 significantly inhibit tumor growth in mice. After 30 days, the tumor volume and weight of AGS-UCA1-KO group were significant smaller and lighter than those of AGS-
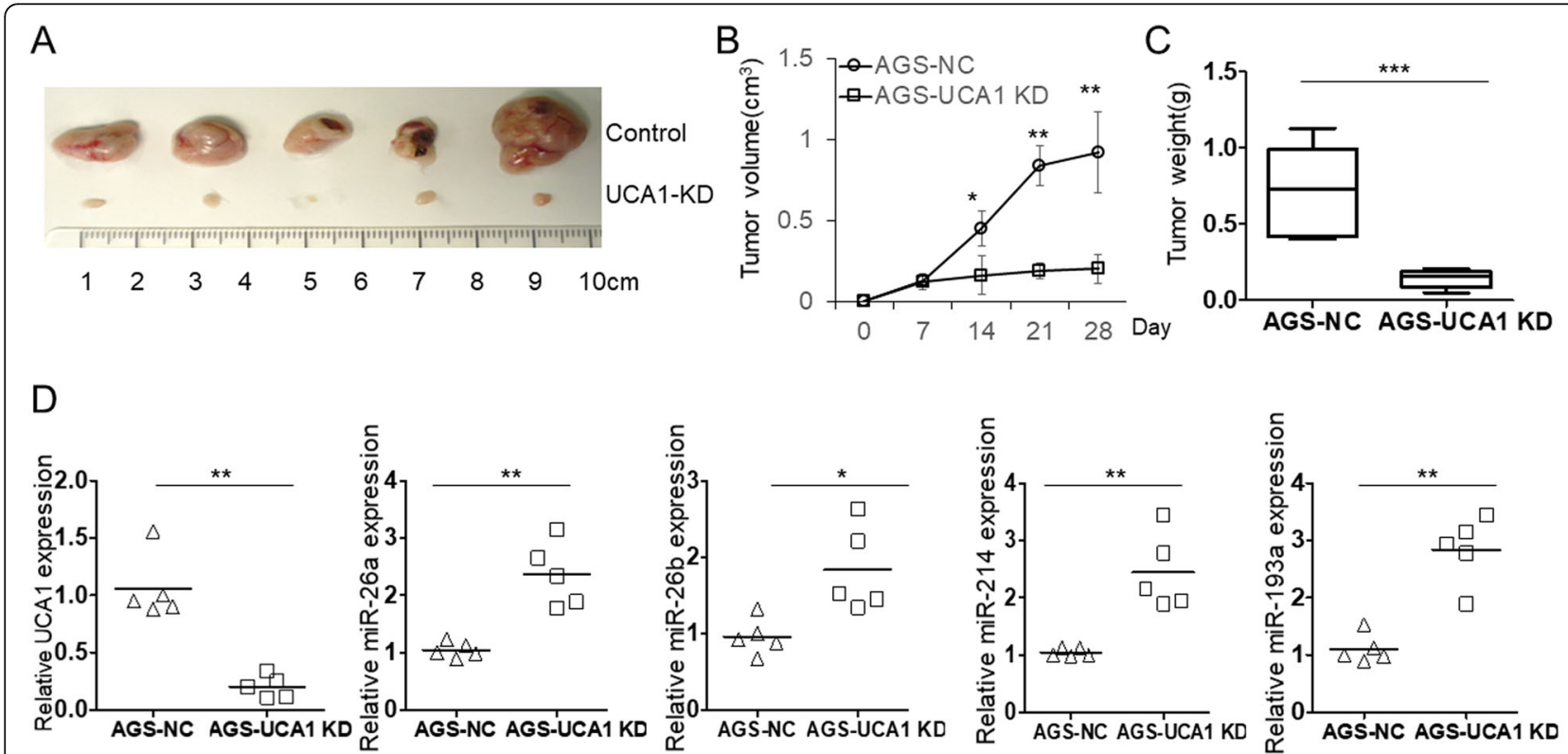

E

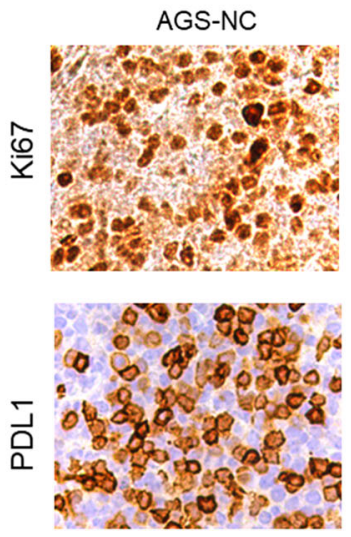

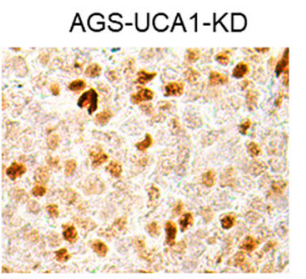

$\mathrm{F}$
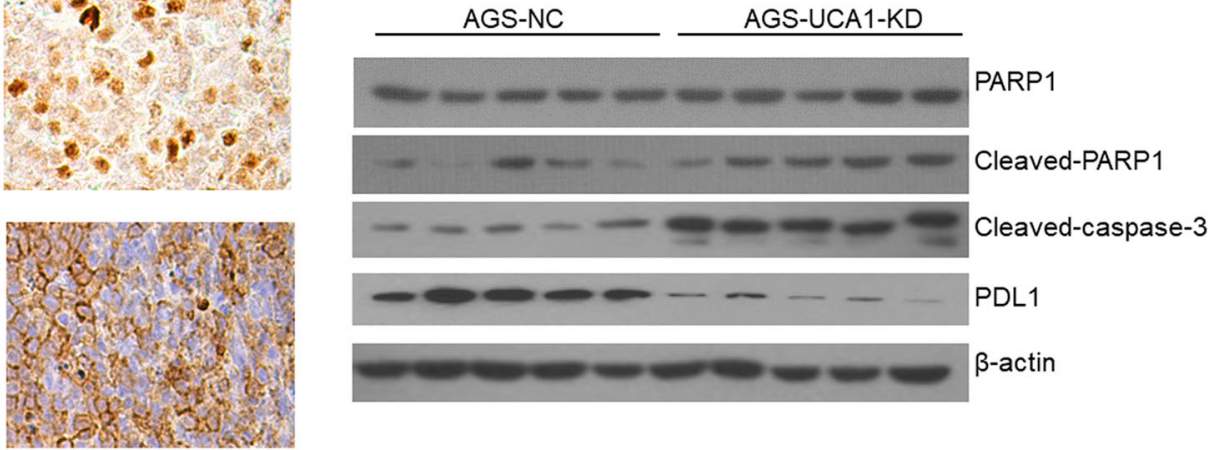

Fig. 6 knockdown of UCA1 expression inhibit the tumor growth in xenograft mouse model. a we examined the effect of knockdown of UCA1 on the regulation of subcutaneous transplantation mouse model of gastric cancer by using AGS cell line. The AGS-NC and AGS-UCA1-KD cells were injected subcutaneously into SCID mice. The tumor volumes were measured every week for 4 weeks. $\mathbf{b}$ the statistical analysis showed that knockdown of PDL1 expression significantly inhibits tumor growth. $\mathbf{c}$ after 28 days, the tumor weight of UCA1-KD group was lighter than that of control group $(p<0.001)$. $\mathbf{d}$ the qRT-PCR results showed that the level of miR-26a, miR-26b, miR-193a and miR-214 was increased in the UCA1-KD group. e the immunohistochemistry results showed that PDL1 protein level as well as Ki-67 was down regulated in UCA1-KD group. $\mathbf{f}$ The immunoblotting results showed that cleaved PARP1 and caspase-3 level was increased in the UCA1-KD tumors 
NC group (Fig. 6b, c). Meanwhile, the level of antitumor miRNAs (miR-26a, miR-26b, miR-193a and miR214) that directly interact with UCA1, was increased in the UCA1-KO tumors (Fig. 6d). Decreased Ki-67, PDL1 and increased cleaved PARP1 and caspase- 3 were also found in the UCA1-KO tumors (Fig. 6e and f).

\section{Discussion}

LncRNAs have been identified to function as key regulators of diverse cellular processes including the initiation and progression of cancer. As an IncRNA, although UCA1 was found overexpressed in gastric cancer, the role of UCA1 in GC is still not fully understood. In this study, we first analyzed two newly released microarray datasets, and found the consistent results showing overexpressed UCA1 in the GC tissues. According to Lauren's criteria, gastric cancer is classified into two major histological subtypes: intestinal type and diffuse type adenocarcinoma [28]. After analyzing the UCA1 level in 40 paired GC and adjacent non-tumor tissue samples, we found that UCA1 especially overexpressed in intestinal GC tissues. Since the two histological subtypes of gastric cancer have lots of distinct clinical and molecular characteristics, further analysis combine the UCA1 expression and other factors including molecular characteristics, drug sensitivity and biological behaviors, should be helpful to guide the GC clinical treatment.

MiR-26a and miR-26b are the two multiple functional miRNAs which were found dysregulated in diverse cancers and are involved in various biological processes, including proliferation, migration, invasion, angiogenesis, and metabolism by modulating different signaling pathways. MiR-26a level has been found to be reduced in the plasma and cancer tissue samples of GC patients, but the mechanism are still not known [29, 30]. We identified that UCA1 functions as a miRNA "sponge" modulating miR-26a and miR-26b level by direct interaction. Furthermore, we also identified the negative correlation between UCA1 level and miR-26a or miR-26b in GC tissue samples, which may provide an answer of how UCA1 modulate the level of miR-26a or miR-26b in GC.

PDL1 is a transmembrane protein that usually overexpressed in cancer cells, and plays a major role in suppressing the immune system by binding with PD1, which was found on activated T cells, B cells, and myeloid cells [31]. In gastric cancer, increasing evidences indicates that upregulated PDL1 level associates cancer progression and worse prognosis [32, 33]. In this study, we identified that miR-193a and-214 repressed PDL1 expression by targeting 3'UTR. Meanwhile, we confirmed that UCA1 functioned as a "miRNA sponge" absorbs miR-193a and miR-214 and protect the PDL1 expression at RNA level. These results indicated that UCA1 targeting treatment may not only a treatment targeting the tumor cells, but also a therapy activates the host immune system. Furthermore, our research indicated that UCA1-KO GC cells could induced a higher IFNY expression when co-cultured with PBMCs, and have a lower survival rate when co-cultured with $\mathrm{CIK}$ cells in vitro, suggesting UCA1-KO GC cells may be more easily to be eliminated by the immune system.

In conclusion, we present here that UCA1 functions as an onco-lncRNA promotes GC cells proliferation, migration and inhibits GC cells apoptosis by repressing antitumor miRNAs as miR-26a and miR-26a. Meanwhile, UCA1 also represses the host immune systems by upregulating the PDL1 level of GC cells. UCA1-KO GC cells formed smaller tumors, and had higher miR-26a, $-26 \mathrm{~b}$, $-193 \mathrm{a}$ and -214 level, reduced cell proliferation and increased apoptosis in vivo, indicating that UCA1 could serve as a potential novel therapeutic target for GC treatment.

\section{Additional files}

Additional file 1: Figure S1. Establish UCA1 knockdown cells. AGS or SGC-7901 cells were co-transfected with pUCA1-KD and plentiCas9-Blast followed by selection for more than 5 days with blasticidin $(4 \mu \mathrm{g} / \mathrm{ml})$ and puromycin $(2 \mu \mathrm{g} / \mathrm{ml})$ for 1 week. The knockout of UCA1 promoter region was confirmed by genotyping and sequencing. (A) Genotyping results. The wildtype PCR product is $1705 \mathrm{bp}$ and the expected bands are around $1103 \mathrm{bp}$. The lanes near the markers are wildtype PCR products. Two successful UCA1 knockdown clones in each cell line were labeled. Nonlabeled lanes were the clones not used for functional study. (B)Sequencing results for UCA1 knockdown AGS clones. (C) Sequencing results for UCA1 knockdown SGC-7901 clones. (TIF 7729 kb)

Additional file 2: Figure S2. UCA1 regulate EZH2 and CDK6 expression dependent on sponging miR-26a/b. (A) immunoblotting quantify EZH2 and CDK6 protein level in tumors and control tissues from patients with intestinal GC. (B) correlation analysis between UCA1 and CDK6 level in tumors. (C) correlation analysis between UCA1 and EZH2 level in tumors. (D) $\mathrm{miR}-26 \mathrm{a} / \mathrm{b}$ antagonists rescued the repressive function of UCA1 siRNAs on EZH2 and CDK6 expression. (TIF $3313 \mathrm{~kb}$ )

Additional file 3: Figure S3. UCA1 interact with miR-26a, miR-26b, miR193a and miR-214 in GC cells and regulate their targets expression. (A) Cell extracts were incubated with biotin labelled UCA1 probe, with biotin labelled sequence scrambled DNA oligo as control. Hybridized material was captured with magnetic streptavidin beads. Beads were subjected to RNA extraction followed by RT-qPCR to quantify miRNAs levels. (B) Fluorescence in situ hybridization to identify the colocalization between UCA1 and miRNAs. (C) miRNAs expressions were quantified by RT-qPCR in UCA1 knockdown cells. (D) The protein level of TAK1(miR-26a/b target), KRAS(miR-193a target) and FGF9 was examined by immunoblotting in UCA1 overexpression or knockdown GC cells (TIF 14 kb)

Additional file 4: Figure S4. miR-193a and miR-214 regulated PDL1 expression and modulated immune response. (A) miR-193a and miR-214 levels were examined by RT-qPCR in tumor and control tissues from patients with GC. The correlation between UCA1 and miRNAs were analyzed. (B) PDL1 protein level was quantified by immunoblotting in tumor and control tissues from patients with GC. The correlation between PDL1 and UCA1 was analyzed. (C) PHA induced PBMCs were co-cultured with miRNA mimic transfected or control GC cells. IFNy production in the supernatant was detected by ELISA. (D) miR-193a and miR-214 mimics transfection improve the cytotoxic sensitivity to CIK therapy in vitro. (TIF 6730 kb)

\section{Abbreviations}

ceRNA: endogenous RNA; CIK: Cytokine-induced killer; FBS: Fetal bovine serum; GC: Gastric cancer; KO: Knock out; IncRNAs: Long non-coding RNAs; 
miRNAs: Micro RNAs; OD: Optical density; PBMCs: Peripheral blood mononuclear cells; SCID: Severe combined immunodeficient; UTR: Untranslated region; WT: Wild type

\section{Acknowledgements}

None.

\section{Authors' contributions}

Guarantor of intergrity of the entire study: ZZZ, G Z. Experimental studies: CJW, JX, CCZ. Data analysis: LT, WYZ, MW. Manuscript preparation: ZZZ, CZ, QL. All authors read and approved the final manuscript.

\section{Funding}

This work is supported by Projects funded by the national natural science foundation of China (31872740), Pudong new area joint project (PW2017D1), The 100-member plan of the Shanghai municipal commission of health and family planning (2017BR043), Shanghai science and technology commission project (17ZR1416800), Shanghai shenkang hospital development center is suitable for technology joint promotion project (SHDC12016236), Renji hospital training fund (PYMDT-003,PYIII-17-015)

\section{Availability of data and materials}

The datasets used and/or analyzed during the current study are available from the corresponding author on reasonable request.

\section{Ethics approval and consent to participate}

Patients and controls were acquired with informed consent, under the protocol approved by Renji Hospital, Shanghai Jiao Tong University School of Medicine research ethics committee.

\section{Consent for publication}

All the authors agree to publish this paper.

\section{Competing interests}

The authors declare that they have no competing interests.

Received: 25 January 2019 Accepted: 22 May 2019

Published online: 04 July 2019

\section{References}

1. Parkin DM, Bray FI, Devesa SS. Cancer burden in the year 2000. The global picture. Eur J Cancer. 2001;37(Suppl 8):S4-66.

2. Parkin DM. International variation. Oncogene. 2004;23:6329-40.

3. Ferlay J, Soerjomataram I, Dikshit R, Eser S, Mathers C, Rebelo M, et al. Cancer incidence and mortality worldwide: sources, methods and major patterns in GLOBOCAN 2012. Int J Cancer. 2015;136:E359-86.

4. Jemal A, Bray F, Center MM, Ferlay J, Ward E, Forman D. Global cancer statistics. CA Cancer J Clin. 2011:61:69-90.

5. Carninci P, Kasukawa T, Katayama S, Gough J, Frith MC, Maeda N, et al. The transcriptional landscape of the mammalian genome. Science. 2005;309: 1559-63.

6. Katayama S, Tomaru Y, Kasukawa T, Waki K, Nakanishi M, Nakamura M, et al. Antisense transcription in the mammalian transcriptome. Science. 2005;309:1564-6.

7. Djebali S, Davis CA, Merkel A, Dobin A, Lassmann T, Mortazavi A, et al. Landscape of transcription in human cells. Nature. 2012;489:101-8.

8. Kung JT, Colognori D, Lee JT. Long noncoding RNAs: past, present, and future. Genetics. 2013;193:651-69.

9. Guan D, Zhang W, Zhang W, Liu GH, Belmonte JC. Switching cell fate, ncRNAs coming to play. Cell Death Dis. 2013;4:e464.

10. Tay Y, Rinn J, Pandolfi PP. The multilayered complexity of ceRNA crosstalk and competition. Nature. 2014;505:344-52.

11. Xia $T$, Liao $Q$, Jiang $X$, Shao $Y$, Xiao $B, X i Y$, et al. Long noncoding RNA associated-competing endogenous RNAs in gastric cancer. Sci Rep. 2014:4:6088.

12. Cao WJ, Wu HL, He BS, Zhang YS, Zhang ZY. Analysis of long non-coding RNA expression profiles in gastric cancer. World J Gastroenterol. 2013;19: 3658-64.

13. Pang $Q$, Ge J, Shao $Y$, Sun $W$, Song $H$, Xia $T$, et al. Increased expression of long intergenic non-coding RNA LINC00152 in gastric cancer and its clinical significance. Tumour Biol. 2014;35:5441-7.
14. Wang XS, Zhang Z, Wang HC, Cai JL, Xu QW, Li MQ, et al. Rapid identification of UCA1 as a very sensitive and specific unique marker for human bladder carcinoma. Clin Cancer Res. 2006;12:4851-8.

15. Zuo ZK, Gong Y, Chen XH, Ye F, Yin ZM, Gong QN, et al. TGFbeta1-induced LncRNA UCA1 upregulation promotes gastric Cancer invasion and migration. DNA Cell Biol. 2017;36:159-67.

16. Shang C, Guo Y, Zhang J, Huang B. Silence of long noncoding RNA UCA1 inhibits malignant proliferation and chemotherapy resistance to adriamycin in gastric cancer. Cancer Chemother Pharmacol. 2016;77:1061-7.

17. Schmidt-Wolf GD, Negrin RS, Schmidt-Wolf IG. Activated T cells and cytokine-induced CD3+CD56+ killer cells. Ann Hematol. 1997;74:51-6.

18. Szasz AM, Lanczky A, Nagy A, Forster S, Hark K, Green JE, et al. Crossvalidation of survival associated biomarkers in gastric cancer using transcriptomic data of 1,065 patients. Oncotarget. 2016;7:49322-33.

19. Bayoumi AS, Sayed A, Broskova Z, Teoh JP, Wilson J, Su H, et al. Crosstalk between long noncoding RNAs and MicroRNAs in health and disease. Int J Mol Sci. 2016;17:356.

20. Zhu Y, Lu Y, Zhang Q, Liu JJ, Li TJ, Yang JR, et al. MicroRNA-26a/b and their host genes cooperate to inhibit the G1/S transition by activating the pRb protein. Nucleic Acids Res. 2012:40:4615-25.

21. Lu J, He ML, Wang L, Chen Y, Liu X, Dong Q, et al. MiR-26a inhibits cell growth and tumorigenesis of nasopharyngeal carcinoma through repression of EZH2. Cancer Res. 2011;71:225-33.

22. Yang TS, Yang XH, Wang XD, Wang YL, Zhou B, Song ZS. MiR-214 regulate gastric cancer cell proliferation, migration and invasion by targeting PTEN. Cancer Cell Int. 2013;13:68

23. Yu T, Li J, Yan M, Liu L, Lin H, Zhao F, et al. MicroRNA-193a-3p and -5p suppress the metastasis of human non-small-cell lung cancer by downregulating the ERBB4/PIK3R3/mTOR/S6K2 signaling pathway. Oncogene. 2015;34:413-23.

24. Dong H, Zhu G, Tamada K, Chen L. B7-H1, a third member of the B7 family, co-stimulates T-cell proliferation and interleukin-10 secretion. Nat Med. 1999:5:1365-9.

25. Ceeraz S, Nowak EC, Noelle RJ. B7 family checkpoint regulators in immune regulation and disease. Trends Immunol. 2013;34:556-63.

26. Guo Y, Yu P, Liu Z, Maimaiti Y, Wang S, Yin X, et al. Prognostic and Clinicopathological value of programmed death Ligand-1 in breast Cancer: a meta-analysis. PLoS One. 2016;11(5):e0156323.

27. Zhang W, Liu J, Qiu J, Fu X, Tang Q, Yang F, et al. MicroRNA-382 inhibits prostate cancer cell proliferation and metastasis through targeting COUPTFII. Oncol Rep. 2016:36:3707-15.

28. van der Woude CJ, Kleibeuker JH, Tiebosch AT, Homan M, Beuving A Jansen $\mathrm{PL}$, et al. Diffuse and intestinal type gastric carcinomas differ in their expression of apoptosis related proteins. J Clin Pathol. 2003:56(9):699-702.

29. Qiu X, Zhu H, Liu S, Tao G, Jin J, Chu H, et al. Expression and prognostic value of microRNA-26a and microRNA-148a in gastric cancer. J Gastroenterol Hepatol. 2017;32:819-27.

30. Qiu X, Zhang J, Shi W, Liu S, Kang M, Chu H, et al. Circulating MicroRNA-26a in plasma and its potential diagnostic value in gastric Cancer. PLoS One. 2016;11:e0151345.

31. Bersanelli M, Buti S. From targeting the tumor to targeting the immune system: transversal challenges in oncology with the inhibition of the PD-1/ PD-L1 axis. World J Clin Oncol. 2017:8:37-53.

32. Liu YX, Wang XS, Wang YF, Hu XC, Yan JQ, Zhang YL, et al. Prognostic significance of PD-L1 expression in patients with gastric cancer in East Asia: a meta-analysis. OncoTargets Ther. 2016;9:2649-54.

33. Tamura T, Ohira M, Tanaka H, Muguruma K, Toyokawa T, Kubo N, et al. Programmed Death-1 Ligand-1 (PDL1) expression is associated with the prognosis of patients with stage II/III gastric Cancer. Anticancer Res. 2015;35: 5369-76.

\section{Publisher's Note}

Springer Nature remains neutral with regard to jurisdictional claims in published maps and institutional affiliations. 